\title{
The Expression of miR-365 in Serum of Hypertension Patients with Left Ventricular Hypertrophy Was Up-Regulated, Which Was Positively Correlated with Left Ventricular Mass Index
}

\author{
Hai-bo $\mathrm{Wu}^{1, *}$ \\ Chen-si Yang ${ }^{1,2, *}$ \\ Yun-can Wang ${ }^{3}$ \\ Yue-tao Xie' \\ Xue-chao Wang' \\ Hui-liang Liu' \\ Rong-pin $\mathrm{Du}^{\prime}$
}

'Department of Cardiology, Hebei General Hospital, Shijiazhuang, Hebei, People's Republic of China; ${ }^{2} \mathrm{Hebei}$ Northen University Graduate School, Zhangjiakou, Hebei, People's Republic of China; ${ }^{3}$ Department of Ultrasound, The First Hospital of Hebei Medical University, Shijiazhuang, Hebei, People's Republic of China

*These authors contributed equally to this work
Correspondence: Rong-pin Du Department of Cardiology, Hebei General Hospital, No. 348, Peace West Road, Shijiazhuang, 050000, Hebei, People's Republic of China

Tel +86 I5I28I38630

Fax +86031185989696

Email durp_asII@I63.com
Objective: The present study aims to investigate micro ribonucleic acid-365 (miR-365) serum expression and its correlation with left ventricular hypertrophy (LVH) in patients with hypertension (HT).

Methods: Eighty-four patients were selected as study subjects and divided into three groups: the experimental group $(n=28)$, the observation group $(n=29)$, and the control group $(n=27)$. The experimental group included patients with LVH-accompanied HT who were treated in the People's Hospital of Hebei Province between November 2019 and November 2020, the observation group included patients with HT unaccompanied by $\mathrm{LVH}$, and the control group included healthy age and gender-matched subjects who underwent health examinations in our physical examination center. The cardiac echocardiography, 24-h Holter electrocardiogram, and circulating miR-365 levels in all subjects were measured. The differences in circulating miR-365 expression levels among the three groups were compared, and the correlations between the miR-365 expression levels and the blood pressure parameters (24-h mean systolic blood pressure [SBP] and 24-h mean diastolic blood pressure [DBP]), inter-ventricular septal thickness (IVST), left ventricular posterior wall thickness (LVPWT), left ventricular internal diameter (LVID), left ventricular mass (LVM), LVM index (LVMI), and LVH-related indicators were analyzed.

Results: The relative miR-365 expressions in the experimental, observation, and control groups were $2.08(1.60,2.34), 0.62(0.44,0.83)$, and $0.66(0.35,0.86)$, respectively. Patient miR-365 expression was significantly higher in the experimental group than in the observation group and the control group; the differences were statistically significant $(p<0.000)$. Furthermore, miR-365 expression was significantly correlated with SBP, DBP, IVST, LVPWT, LVID, LVM, and LVMI; the greatest correlation was with LVMI. Further univariate linear regression analysis revealed that miR-365 expression was linearly and positively correlated with LVMI and that miRNA-365 expression increased with the LVMI value.

Conclusion: The miR-365 serum expression in patients with LVH-accompanied HT was increased compared with the observation group and the control group and positively correlated with the LVH degree.

Keywords: essential hypertension, left ventricular hypertrophy, RT-PCR, microRNA

\section{Introduction}

The increasing incidence of cardiovascular disease (CVD) has become a major public health challenge not only in Western, but also Asian countries (where it is prominent). ${ }^{1}$ Hypertension (HT) and left ventricular hypertrophy (LVH) are both independent risk 
factors for CVD development. ${ }^{2}$ Myocardial hypertrophy $(\mathrm{MH})$, one of the common CVD pathological features, is divided into two categories: physiological and pathological. The majority of clinically diagnosed patients have developed pathological $\mathrm{MH}$, which refers to an injury response to longterm mechanical stimulation, endocrine factor changes, or metabolic disorders that overload the heart and manifests primarily as increased myocardial cell volume; protein synthesis; embryonic gene re-expression; myocytes; and the proliferation of interstitial cells, collagen, and other connective tissues. ${ }^{3}$ Persistent pathological $\mathrm{MH}$ is prone to causing reduced contractility, inadequate blood supply, myocardial systolic and diastolic insufficiency, and myocardial structural disorders. Eventually, it can lead to heart failure, arrhythmias, or even sudden death. ${ }^{4}$ In China, there is a large population with HT, which is the most important cause of LVH. ${ }^{3}$ During clinical practice, $>1 / 3$ of patients with HT also have LVH. Therefore, early hypertensive LVH detection and diagnosis could potentially be powerful measures for early treatment lesion reversion. Micro ribonucleic acids (miRNAs) are a class of endogenous single-stranded, non-coding RNAs with a length of 19-23 nt that target multiple downstream target genes and can regulate cellular genes at the posttranscriptional level by binding to the $3^{\prime}$-untranslated region of the downstream target genes, allowing them to regulate the post-transcriptional cellular gene levels. ${ }^{5,6}$ They then participate in cell proliferation, differentiation, invasion, migration, and signal transduction processes. ${ }^{5}$ MiR-365 is a multifunctional, non-coding small RNA associated with various diseases, and may play an important role in the development of human placenta and the protection of immunity in semi-homologous embryos. Mechanical stress and proinflammatory responses as potential therapeutic targets for osteoarthritis are mediated by miR-365. In addition, ectopic expression of miR-365 may also be a novel diagnostic or prognostic biomarker for different types of cancer. For example, in invasive ductal adenocarcinoma of the pancreas, miR-365 levels are upregulated to enhance pancreatic cancer cell resistance to gemcitabine. However, in patients with NSCLC, down-regulated miR-365 was strongly associated with poor differentiation, advanced TNM staging, and lymph node metastasis. The low expression of miR-365 in malignant melanoma is closely related to lymph node metastasis status and clinical stage. MiR-365 inhifies the growth, invasion and metastasis of malignant melanoma by targeting nerve hair cell protein 1 (NRP 1). ${ }^{7}$ Studies have shown that miR-365 is under-expressed in both human gastric cancer tissues and mouse gastric cancer models. In addition, the overexpression of miR-365 can significantly inhibit the proliferation and tumorigenicity of gastric cancer cells. Other studies have shown that miR-365 is highly expressed in breast cancer tissues, and miR-365 is related to TNM staging of breast cancer. By down-regulating the expression of miR365 , the proliferation and invasion ability of breast cancer cells can be inhibited. ${ }^{8}$ MiR-365 may prove to be a novel prognostic biomarker for GBC patients by directly targeting Rac1 to inhibit the growth, metastasis, and self-renewal of GBC cells. ${ }^{9}$ MiR-365 regulates the BDNF-TrkB signaling axis in STZ-induced DN fibrosis and renal function. ${ }^{10} \mathrm{MiR}$ 365 inhibited proliferation, migration, and invasion by directly binding to the $3^{\prime}$-UTR of ADAM 10 mRNA in TNBC. It is suggested that miR-365/ADAM10 axis may provide a new target for the treatment of breast cancer. ${ }^{11}$ As an important tumor suppressor, miR-365 inhibits cell invasion, proliferation and self-renewal in cancer cell lines by regulating a variety of oncogenes. The identification of miR365-EZH2/Fos pathway is an important target for the treatment of endometrial cancer. ${ }^{12}$

Studies have shown that miR-365 expression is upregulated in hypertrophic cardiomyocytes in vivo and in vitro, accompanied by autophagy dysregulation. We found that miR-365 negatively regulates autophagy in hypertrophic cardiomyocytes by targeting Skp2. Skp2 overexpression promotes autophagy and rescents Ang-II-induced CH; In contrast, Skp2 knockdown further inhibited autophagy and $\mathrm{CH}^{13}$ In addition, correlation analysis showed that miR$30 \mathrm{~b}$ and miR-30e expression levels were positively correlated with left ventricular mass index (LVMI), respectively. Moreover, we found that circulating miR-30e level can be used to differentiate essential hypertension-left ventricular hypertrophy. EH-LVH) and essential hypertension-non-left ventricular hypertrophy (EH-NLVH). It has been tentatively suggested that circulating miR-30e predicts left ventricular hypertrophy in patients with essential hypertension. Therefore, it is clear that miRNA is involved in the occurrence and development of cardiac hypertrophy, and it is suggested that miRNA is closely related to the occurrence of cardiac hypertrophy. ${ }^{14}$

A generally accepted concept is that abnormal miRNA expression or function can mediate pathological $\mathrm{MH}^{6-8}$ miRNA-365 (miR-365) is an important member of the miRNA family, and studies have shown that it also plays a crucial role in pathological MH progression. By screening miRNA expression profile changes, Jentzsch et $\mathrm{al}^{9}$ found that miR-365 had a high expression level and was positively correlated with the hypertrophied myocardium 
MH degree, suggesting that miR-365 might have prohypertrophic effects. However, only fundamental and animal experiments have been conducted, and clinical trials have not yet been implemented into studies. The expression level of miR-365 in patients with pathological cardiac hypertrophy and whether it mediates the occurrence and development of cardiac hypertrophy are still unclear. ${ }^{10,11}$ As an independent risk factor for heart failure, acute myocardial infarction, stroke, and sudden cardiac death occurrence, $\mathrm{MH}$ pathogenesis needs to be further investigated. This is a key aspect to clinical prevention, treatment, and prognosis improvement. Hence, the present study aims to investigate miR-365 serum expression and its correlation with $\mathrm{LVH}$ expression and indicators in patients with LVH-accompanied HT. This should provide a certain level of guidance for future hypertensive LVH diagnosis, prediction, and treatment.

\section{Subjects and Methods \\ Study Subjects}

Eighty-four patients were selected as study subjects and divided into three groups: the experimental group (11 males and 17 females, $n=28)$, the observation group (14 males and 15 females, $\mathrm{n}=29$ ), and the control group (11 males and 16 females, $n=27)$. The experimental group included patients with LVH-accompanied HT who were treated in the People's Hospital of Hebei Province between November 2019 and November 2020, with an average age of $51.14 \pm 13.02$ years; the observation group included patients with HT unaccompanied by LVH, with an average age of $52.62 \pm 9.85$ years; and the control group included healthy age and gender-matched subjects who underwent health examinations in our physical examination center, with an average age of $47.85 \pm 12.73$.

Subjects in the control group had no previous history of pulmonary embolism, malignancy, surgery within the last 6 weeks, cardiac insufficiency, or a cerebrovascular accident. This study was conducted with approval from the Ethics Committee of Hebei People's Hospital (No.: 2018KY147). This study was conducted in accordance with the declaration of Helsinki. All patients signed an informed consent form.

\section{Inclusion and Exclusion Criteria}

Inclusion criteria: (1) patients with new-onset essential HT or patients diagnosed with essential HT who had developed LVH without antihypertensive medication; (2) patients with essential HT unaccompanied by LVH who were not taking medication; (3) healthy physical examination subjects (with reference to the English version of the 2018 Chinese Guidelines for the Prevention and Treatment of HT's diagnostic criteria). ${ }^{12}$

Exclusion criteria: (1) patients with hypertrophic or constrictive cardiomyopathy; (2) patients with secondary HT; (3) patients with HT with present or past use of antihypertensive drugs; (4) patients with HT combined with coronary artery disease, diabetes mellitus, malignant arrhythmia, moderate-severe valvular disease, congenital heart disease, hyperthyroidism, hypothyroidism, hepatic and renal insufficiency, heart failure, or tumors; (5) smokers; (6) patients who did not attend follow-up visits and were unwilling to sign the informed consent form.

\section{Methods}

\section{Blood Sample Collection and Preparation}

In the early morning of the second day of enrollment, 2$4 \mathrm{~mL}$ of venous blood was drawn from the forearm after fasting in all patients and put into a red sterile collection tube. Each sample was centrifuged in our clinical laboratory research center for $10 \mathrm{~min}$ at $3000 \mathrm{r} / \mathrm{min}$ and $4^{\circ} \mathrm{C}$. The specimen serum was then stored in a sterile collection tube without an RNA enzyme using a sterile pipette. All collected specimens were transferred to $\mathrm{a}-80^{\circ} \mathrm{C}$ refrigerator, for storage and subsequent experiments, within $2 \mathrm{~h}$.

\section{Hypertension Diagnostic Criteria}

The following process was conducted with reference to the HT diagnostic criteria according to the English version of the 2018 Chinese Guidelines for the Prevention and Treatment of HT. ${ }^{12}$ Without the application of antihypertensive drugs, patients with systolic blood pressure (SBP) $\geq 140 \mathrm{mmHg}$ and/or diastolic blood pressure (DBP) $\geq 90$ $\mathrm{mmHg}$ measured on three office visits on various days were diagnosed with HT. Patients with SBP $\geq 140$ $\mathrm{mmHg}$ and $\mathrm{DBP}<90 \mathrm{mmHg}$ were considered to have simple systolic HT. Morning and evening blood pressure as well as 24-hour ambulatory blood pressure measurements were conducted in all the enrolled subjects. The subjects were in a quiet lying position during the procedure, and the data were recorded to the medical record.

\section{Echocardiographic Testing}

The left ventricular mass index (LVMI) is the main echocardiography-detected LVH indicator. ${ }^{13}$ M-mode 
echocardiography is most commonly used in clinical practice. In each enrolled subject, cardiac ultrasonography measurements were completed and the left ventricular internal diameter (LVID), interventricular septal thickness (IVST), and left ventricular posterior wall thickness (LVPWT) were measured using an ultra-sonographer in accordance with the American Society of Echocardiography recommended methods. Next, the left ventricular mass (LVM) was calculated as follows:

$$
\begin{aligned}
& \mathrm{LVM}(\mathrm{g})=1.04 \times\left[\left(\mathrm{LVID}+\mathrm{IVST}+\mathrm{LVPWT}^{3}-\mathrm{LVID}^{3}\right]\right. \\
& \quad \times 0.8+0.6
\end{aligned}
$$

In accordance with the Devereux correction formula, LVMI was obtained after LVM correction by the body surface area (BSA) (ie LVMI $\left[\mathrm{g} / \mathrm{m}^{2}\right]=\mathrm{LVM} / \mathrm{BSA}$ ).

The LVH diagnosis criteria evaluated using the LVMI were $\geq 115 \mathrm{~g} / \mathrm{m}^{2}$ in males and $\geq 95 \mathrm{~g} / \mathrm{m}^{2}$ in females.

\section{Experimental Steps of the Reverse} Transcription-Polymerase Chain Reaction The total serum sample RNA was extracted using the Trizol method and quantified using the spectrophotometer. The quality of total RNA was then determined using formaldehyde denaturing gel electrophoresis. ${ }^{14-16}$ Reagents used were: MiCute miRNA Extraction and Isolation Kit (DP501), (Tien Root Biochemistry), RNase free suction head (Axygen), RNASE Free EP Tube (Axygen), Electrophoresis apparatus (Beijing Junyi), Automatic Gel Imaging Analysis System (Shanghai Peiqing), Frozen Centrifuge (Zhuhai Black Horse), Eight header (Axygen), MiCute Enhanced First strand miRNA cDNA Synthesis Kit (KR211) (Tien Root Biochemistry), MiCute Enhanced MicroRNA Fluorescence Quantitative Assay Kit (SYBR Green)(FP411) (Tien Root Biochemistry), Real-time fluorescence quantitative PCR instrument (ABI 7500).

(1) All samples were centrifuged, and the serum was taken from the blood and stored at $-80^{\circ} \mathrm{C}$ in our clinical research center. First, the samples were taken out for RNA extraction; second, direct reverse transcription was performed in order to form stable complementary deoxyribonucleic acid (cDNA).

The first cDNA chain synthesis: reagents including $8 \mu \mathrm{L}$ of total RNA, $10 \mu \mathrm{L}$ of $2 \times$ miRNA reverse transcription (RT) reaction buffer, and $2 \mu \mathrm{L}$ of miRNA RT enzyme mix were added to a total volume of $20 \mu \mathrm{L}$ in the ribonuclease free reaction tube, which was pre-cooled on ice, and mixed well.
(2) After the above-listed reagents were thoroughly mixed, the reaction was carried out for $60 \mathrm{~min}$ at $42^{\circ} \mathrm{C}$. Next, the reaction tube was heated for $3 \mathrm{~min}$ at $95^{\circ} \mathrm{C}$, inactivating the reverse transcriptase and denaturing the RNA-cDNA heterodimer. The reaction solution was then stored at $-20^{\circ} \mathrm{C}$ for use in further experiments.

(3) Polymerase chain reaction (PCR) preparation: with the reverse transcription completion, each sample was subjected to quantitative PCR using the same tube of cDNA but different primers. The u6 RNA was adopted as the internal reference. The PCR reaction conditions were as follows: $15 \mathrm{~min}$ at $95^{\circ} \mathrm{C}$ for one cycle and $20 \mathrm{~s}$ at $94^{\circ} \mathrm{C}+34 \mathrm{~s}$ at $60^{\circ} \mathrm{C}$ for 40 cycles.

\section{Data Processing: The $2^{-\Delta c t}$ Method Was Adopted for the Fluorescent Quantitative PCR Data Analysis}

The $2^{-\Delta \mathrm{Ct}}$ method: (1) those with a $\mathrm{Ct}$ value of $>35$ were deleted, and the average (AVG) value of the remaining compound holes was evaluated; (2) the $\Delta \mathrm{Ct}, \Delta \mathrm{Ct}=\mathrm{AVG}$ of the detected miRNA, and the AVG of the internal reference gene were calculated; and (3) the multiplicative change $\left(2^{-\Delta \mathrm{Ct}}\right)$ was calculated.

* All tested sample gene expressions were shown as relative expressions and compared using the $2^{-\Delta \mathrm{Ct}}$ method.

\section{Statistical Analysis}

The Statistical Package for Social Sciences 26.0 software was adopted for data analysis. Measurement data that conformed to a normal distribution were expressed as a mean \pm standard deviation, and measurement data that did not conform to a normal distribution were expressed in quartiles. The ranksum test (Kruskal-Wallis H method) of k independent samples was used to analyze the general patient group characteristics. The expression differences between the groups were further analyzed using the two-by-two multiple sample comparison. The Spearman correlation analysis was adopted to analyze the correlation between miR-365 expression and IVST, LVPWT, LVID, LVM, LVMI, SBP, and DBP expressions. Finally, linear regression was established in order to represent the linear relationship between miR-365 and hypertensive LVH in the subjects.

\section{Results}

\section{Baseline Data Comparison Among the Three Groups}

In the present study, the experimental group included 28 patients (11 males and 17 females) with LVH- 
accompanied HT, the observation group included 29 patients (14 males and 15 females) with HT unaccompanied by LVH, and the control group included 28 healthy physical examination subjects (11 males and 16 females). There was no statistical significance in the distribution of gender among different groups $(\chi 2=0.543, \mathrm{P}=0.762)$, and the inclusion was more balanced.

There was no statistically significant difference in gender distribution among the three groups $\left(\chi^{2}=0.543, p=0.762\right)$.

There was no statistically significant difference in age among the three groups $(\mathrm{F}=1.163, \mathrm{p}=0.318)$ (Table 1$)$; however, there was a statistically significant difference in SBP $(F=100.823, p=0.000)$. Further two-by-two least significant Difference (LSD) comparison showed that the SBP levels were lower in the control group than in the experimental group $(124.52 \mathrm{mmHg}<164.71 \mathrm{mmHg}, \mathrm{p}<0.05)$, lower in the control group than in the observation group (124.52 $\mathrm{mmHg}<158.66 \mathrm{mmHg}, \mathrm{p}<0.05)$, and lower in the observation group than that in the experimental group (158.66 $\mathrm{mmHg}<164.71 \mathrm{mmHg}, \mathrm{p}<0.05)$.

The results showed that there were statistically significant differences in miR-365, LVM, LVMI, IVST, LVPWT, LVID, BMI, and DBP expressions among the groups $(\mathrm{p}<0.05)$ (Table 2). Further multiple comparisons showed that the miRNA365, LVM, LVMI, IVST, LVPWT, and LVID expressions were lower in the control group and the observation group than in the experimental group $(\mathrm{p}<0.05)$. There was no statistically significant difference in the above-listed indicators between the observation group and the control group. DBP was statistically different among the three groups in the two-by-two comparisons; it was lower in the control group than in the experimental group $(76.00 \mathrm{mmHg}<$ $95.00 \mathrm{mmHg}, \mathrm{p}<0.05)$, lower in the control group than in the observation group (76.00 $\mathrm{mmHg}<90.00 \mathrm{mmHg}, \mathrm{p}<0.05)$, and lower in the observation group than in the experimental group $(90.00 \mathrm{mmHg}<95.00 \mathrm{mmHg}, \mathrm{p}<0.05)$.

\section{RT-PCR-Detected miR-365 Expression Results}

The miR-365 expressions were determined using the RTPCR; the results showed that the relative miR-365 expressions in the experimental, observation, and control groups were $2.08(1.60,2.34), 0.62(0.44,0.83)$, and $0.66(0.35$, 0.86 ), respectively. The differences among the three groups were statistically significant $(\mathrm{p}<0.000)$ (Figure 1 ).

\section{Results of miR-365 Correlation with Blood Pressure and LVH Analysis}

The Spearman correlation analysis was used to further analyze miR-365 correlation with blood pressure and LVH-related indicators; the results showed that miR-365 was significantly correlated with SBP, DBP, IVST, LVPWT, LVID, LVM, and LVMI levels (Table 3).

Specifically, miR-365 was positively correlated with LVM ( $\mathrm{rs}=0.618, \mathrm{p}=0.000)$, LVMI $(\mathrm{rs}=0.654, \mathrm{p}=$

Table I Comparison of Sex Between the Three Groups

\begin{tabular}{|l|c|c|c|c|c|}
\hline Groups & Experimental Group & Observation Group & Control Group & F & P \\
\hline Age & $51.14 \pm \mid 3.02$ & $52.62 \pm 9.85$ & $47.85 \pm 12.73$ & 1.163 & 0.318 \\
SBP & $164.71 \pm \mid 3.21$ & $158.66 \pm 10.82^{\mathrm{a}}$ & $124.52 \pm\left. 9.5\right|^{\mathrm{ab}}$ & 100.823 & 0.000 \\
\hline
\end{tabular}

Notes: ${ }^{a}$ the difference was statistically significant compared with the experimental group $(P<0.05),{ }^{b}$ the difference was statistically significant compared with the observation group $(P<0.05)$.

Table 2 Comparison of Age and Systolic Blood Pressure Among the Three Groups

\begin{tabular}{|l|c|c|c|c|c|}
\hline Groups & Experimental Group & Observation Group & Control Group & $\chi^{2}$ & $\mathbf{P}$ \\
\hline miR-365 & $2.08(1.60,2.34)$ & $0.62(0.44,0.83)^{\mathrm{a}}$ & $0.66(0.34,0.86)^{\mathrm{a}}$ & 55.408 & 0.000 \\
LVM & $178.90(158.82,213.89)$ & $127.66(111.73,145.49)^{\mathrm{a}}$ & $131.20(118.58,137.77)^{\mathrm{a}}$ & 46.709 & 0.000 \\
LVMI & $107.98(95.58,117.61)$ & $76.42(65.52,84.49)^{\mathrm{a}}$ & $77.93(70.22,80.85)^{\mathrm{a}}$ & 55.345 & 0.000 \\
IVST & $10.00(10.00-11.00)$ & $9.00(8.00,9.00)^{\mathrm{a}}$ & $9.00(8.00,9.00)^{\mathrm{a}}$ & 42.956 & 0.000 \\
LVPWT & $11.00(10.00-11.00)$ & $9.00(8.00,9.50)^{\mathrm{a}}$ & $9.00(8.00,10.00)^{\mathrm{a}}$ & 31.413 & 0.000 \\
LVID & $49.00(46.25,5 I .00)$ & $45.00(45.00,46.00)^{\mathrm{a}}$ & $45.00(43.00,46.00)^{\mathrm{a}}$ & 26.579 & 0.000 \\
BMI & $25.21(23.51,26.31)$ & $25.25(24.24,25.81)$ & $26.30(25.22,28.69)$ & 7.018 & 0.030 \\
DBP & $95.00(92.25,105.25)$ & $90.00(79.00,95.00)^{\mathrm{a}}$ & $76.00(73.00,84.00)^{\mathrm{ab}}$ & 35.793 & 0.000 \\
\hline
\end{tabular}

Notes: athe difference was statistically significant compared with the experimental group $(P<0.05)$, ${ }^{b}$ the difference was statistically significant compared with the observation group $(P<0.05)$. 


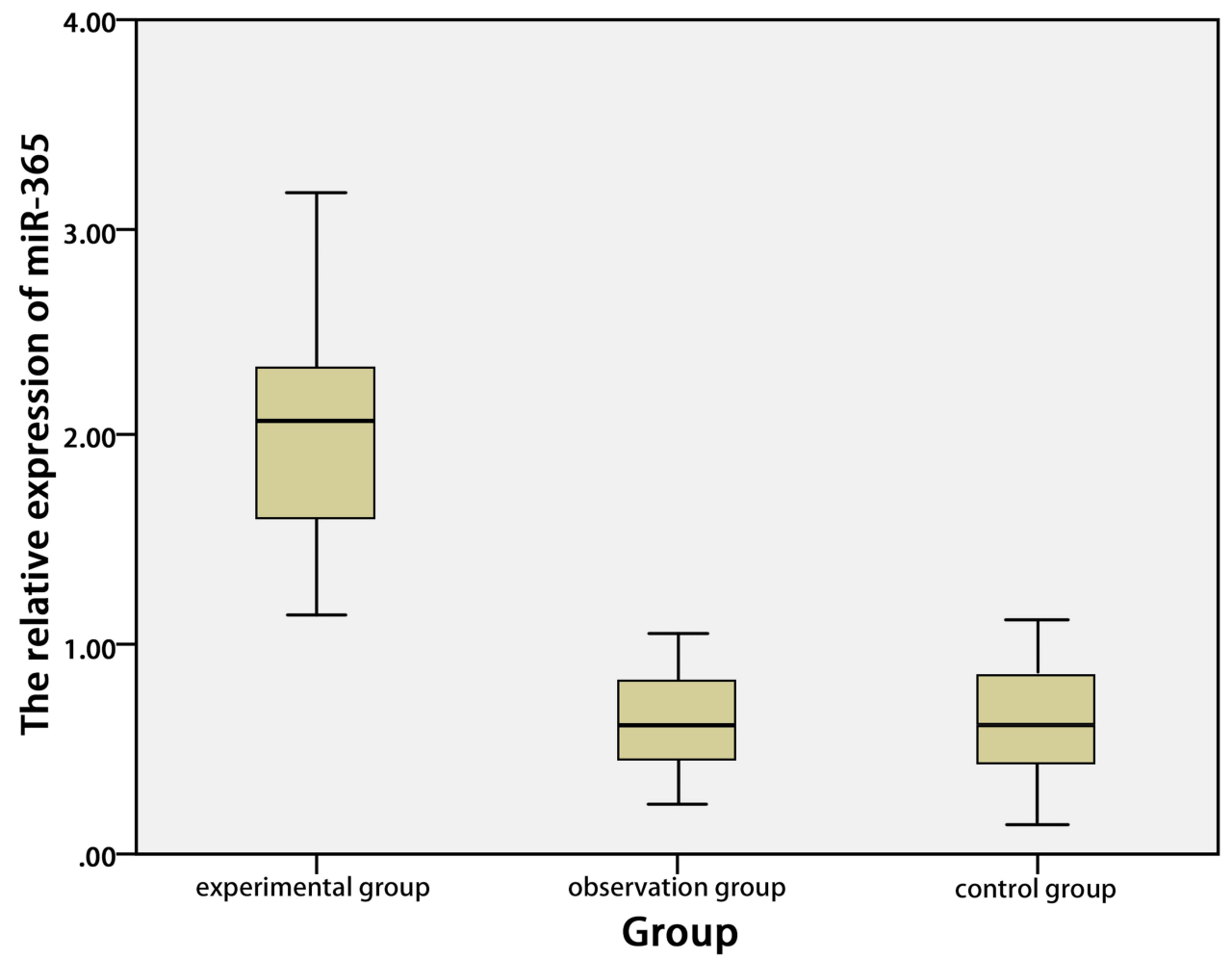

Figure I The expression of miR-365 was measured by RT-PCR. The miR-365 expressions were determined using the RT-PCR; the results showed that the relative miR-365 expressions in the experimental, observation, and control groups were $2.08(1.60,2.34), 0.62(0.44,0.83)$, and $0.66(0.35$, 0.86$)$, respectively. The differences among the three groups were statistically significant $(p<0.000)$.

$0.000), \operatorname{IVST}(\mathrm{rs}=0.623, \mathrm{p}=0.000), \operatorname{LVPWT}(\mathrm{rs}=0.451$, $\mathrm{p}=0.000)$, LVID ( $\mathrm{rs}=0.450, \mathrm{p}=0.000)$, SBP ( $\mathrm{rs}=0.411$, $\mathrm{p}=0.000)$, and DBP ( $\mathrm{rs}=0.460, \mathrm{p}=0.000)($ Table 3$)$. A comprehensive analysis showed that miR-365 expression was primarily correlated with LVMI.

\section{Linear miRNA-365 and LVMI Regressions} Further miR-365 and LVMI regression analysis showed a linear positive correlation between miR-365 expression and LVMI, in which the LVMI was the independent variable and miR-365 was the dependent variable. The higher the LVMI, the higher the relative miR-365 expression. The results are demonstrated in Table 4. The relative miR-365 level increased with the LVMI value when comparing both the experimental group with the observation group and the experimental group with the control group.

\section{Discussion}

LVH is a phenomenon of myocardial changes with ventricular wall thickening, myocardial weight increase, and myocardial remodeling, which is a common hypertension complication $^{14}$ and organ injury target in patients with HT. With HT development, blood pressure fluctuations have a continuous impact on the myocardium. Long-term impact can cause hypertrophic growth response, leading to cell enlargement, enhanced protein synthesis, myogenic fiber aggregation, and embryonic gene reactivation, which can in turn lead to myocardial remodeling and, finally, heart failure or even sudden death. ${ }^{15} \mathrm{LVH}$ is an independent risk factor for heart failure development, acute myocardial infarction, stroke, and sudden cardiac death. ${ }^{19}$

The pathogenesis of HT-induced LVH is complex, and relevant studies in molecular biology have greatly

Table 3 Comparison of miRNA365 and Left Ventricular Hypertrophy Related Indicators Among the Three Groups

\begin{tabular}{|l|l|c|c|c|c|c|c|c|}
\hline & & IVST & LVPWT & LVID & SBP & DBP & LVMI & LVM \\
\hline miR-365 & rs & 0.623 & $0.45 I$ & 0.450 & 0.411 & 0.460 & 0.654 & 0.618 \\
& P & 0.000 & 0.009 & 0.000 & 0.000 & 0.001 & 0.000 & 0.000 \\
\hline
\end{tabular}


Table 4 Linear Regression Analysis of miR-365 and LVWI in Experimental Group, Control Group and Observation Group

\begin{tabular}{|c|c|c|c|c|c|c|c|c|}
\hline & & \multicolumn{2}{|c|}{$\begin{array}{c}\text { Nonstandardized } \\
\text { Coefficient }\end{array}$} & \multirow{2}{*}{$\begin{array}{c}\begin{array}{c}\text { Standardization } \\
\text { Coefficient }\end{array} \\
\text { Beta }\end{array}$} & \multirow[t]{2}{*}{$\mathbf{t}$} & \multirow[t]{2}{*}{ Significance } & \multicolumn{2}{|c|}{$95.0 \% \mathrm{Cl}$} \\
\hline & & B & $\begin{array}{l}\text { Standard } \\
\text { Error }\end{array}$ & & & & $\begin{array}{l}\text { Lower } \\
\text { Limit }\end{array}$ & $\begin{array}{l}\text { Upper } \\
\text { Limit }\end{array}$ \\
\hline \multirow[t]{2}{*}{ LVWI } & Experimental VS Control & 0.029 & 0.003 & 0.769 & 8.747 & 0.000 & 0.023 & 0.036 \\
\hline & Experimental VS Observation & 0.028 & 0.003 & 0.742 & 8.207 & 0.000 & 0.021 & 0.034 \\
\hline
\end{tabular}

facilitated further exploration of miRNAs as candidate biomarkers. The idea that abnormal miRNA expression or function can mediate pathological $\mathrm{MH}$ is now generally accepted. Since their discovery, miRNAs have been a frontier area and a popular subject in molecular biology research. Many miRNA-related studies are reported every year, and the mechanisms and roles of new miRNAs are constantly under further study and exploration. As an important member of the miRNA family, miR365 has been previously focused on by researchers in studies on oncological diseases, and it is believed that miR-365 is primarily involved in malignant tumor development. ${ }^{16}$ It is known that miRNA distribution and expression differ in different tissues and organs (ie, miRNAs are tissue-specific), ${ }^{17}$ and it is also recognized that miR-365 plays an important role in the progression of pathological MH. By screening miRNA expression profile changes, Jentzsch et $\mathrm{al}^{9}$ found that miR-365 was highly expressed in hypertrophied myocardium and that it was positively correlated with the $\mathrm{MH}$ degree, suggesting that miR-365 might have pro-hypertrophic effects. HaiboWu et $\mathrm{al}^{18}$ also found that myocardial miR-365 expression was up-regulated in both in vivo (pathological cardiac hypertrophy rat model) and in vitro (murine hypertrophic cardiomyocytes) and was accompanied by cardiomyocyte autophagy dysregulation. ${ }^{18}$ Further studies revealed that miR-365 may regulate the cardiomyocyte autophagy by targeting the S-phase kinase-associated protein 2, which in turn mediates $\mathrm{MH} .{ }^{18}$ The results of the present study's rigorous clinical trial showed that miR-365 serum expression was up-regulated in patients with LVH-accompanied HT. It further elucidated the correlation of miR-365 with blood pressure and LVH-related indicators, suggesting a clear correlation between miR-365 and LVH.

As for the molecular biological exploration of the pathogenesis of hypertension, some studies have shown that the occurrence of EH (essential hypertension) is closely related to miRNA, ${ }^{17,18}$ and miRNA plays a key role in the occurrence and development of EH. Recent results from a 5-year longitudinal study confirm the association between miRNAs in circulating blood vessels and hypertension, ${ }^{19}$ suggesting that low expression levels of miR-126, miR-221 and miR-222 in serum are associated with increased blood pressure and new episodes of hypertension. In addition, other studies ${ }^{20}$ found significant differences in miR-3135b and miR-107 expression between severe hypertension and healthy controls, and further revealed significant differences in target genes independently regulated by these two miRNAs, which further increased people's exploration on the mechanism of differential expression of circulating miRNAs. The difference in the expression of circulating miRNA between hypertensive patients and normal controls suggests that the expression of miRNA may provide a new direction for the prediction and diagnosis of hypertension, and may be further explored as a potential biomarker for the prediction of hypertension. However, no studies have reported the direct correlation between miR-365 and blood pressure. The differences in blood pressure between groups in this paper (possibly due to small sample size, measurement error and other factors) may interfere with the judgment of the upregulation of miR-365 in serum of patients with hypertension and left ventricular hypertrophy.

At present, the exploration of miRNAs as biomarkers is still in the initial stage, and the monitoring of miRNA levels in tissues provides a new biomarker for clinical disease prediction and diagnosis. Further exploration of gene therapy and target intervention to restore the disordered miRNA levels will provide new disease diagnostic and treatment ideas. However, the sample size in the present study is small, with factors such as different disease developmental stages and etiologies in patients, and further large-sample, multicenter, randomized controlled studies are yet to be conducted. Moreover, the statistical differences in SBP among the study groups may have affected the correlation analysis of blood 
pressure and miR-365 expression. Therefore, to further explore the diagnostic and predictive value of miR-365 in $\mathrm{LVH}$, the sample size should be expanded in order to reduce the individual differences and the application value of miR-365 in LVH-accompanied HT should be further explored.

\section{Conclusion}

Subjects in the present study were enrolled in strict accordance with the inclusion and exclusion criteria, and the patient grouping was clear. There were no statistically significant differences between the experimental, observation, and control group in terms of general clinical data, such as gender and age, which ensured the rigor and reliability of the present study. However, the diagnostic and predictive value of miR-365 in LVH-accompanied HT was not further explored due to the small sample size, individual differences, and different patient developmental stages and etiologies. Large-sample, multicenter, randomized controlled studies are still to be conducted.

It could be inferred from the comprehensive analysis that the miR-365 serum expression was significantly upregulated in patients with LVH-accompanied HT and that the expression difference was significantly correlated with blood pressure, IVST, LVPWT, LVID, LVM, and LVMI, the strongest correlation being with LVMI. Univariate linear regression analysis revealed that the relative miR-365 expression increased with the increase of LVMI, further clarifying the correlation between miR365 expression and LVH. With high sensitivity and low error in the assay, miR-365 is an effective new biomarker for clinical translational study. It is expected to be a novel biomarker for patients with essential LVHaccompanied HT, which would help in exploring the new therapeutic clinical disease strategies, slowing disease progression, and improving prognosis.

\section{Ethics Approval and Consent to Participate}

This study was conducted with approval from the Ethics Committee of Hebei People's Hospital (No.: 2018-KY147). This study was conducted in accordance with the declaration of Helsinki. All patients signed an informed consent form.

\section{Funding}

Natural Science Foundation of Hebei Province (No: H2019307088).

\section{Disclosure}

The authors declare that they have no competing interests.

\section{References}

1. Park SK, Oh CM, Kang JG, et al. The association between left ventricular hypertrophy and consumption of nuts, including peanuts, pine nuts, and almonds. Nutr Metab Cardiovasc Dis. 2021;31 (1):76-84. doi:10.1016/j.numecd.2020.08.009

2. Bang CN, Soliman EZ, Simpson LM, et al. Electrocardiographic Left Ventricular Hypertrophy Predicts Cardiovascular Morbidity and Mortality in Hypertensive Patients: the ALLHAT Study. Am J Hypertens. 2017;30(9):914-922. doi:10.1093/ajh/hpx067

3. Yu F, Huang H, Yu Q, et al. Artificial intelligence-based myocardial texture analysis in etiological differentiation of left ventricular hypertrophy. Ann Transl Med. 2021;9(2):108. doi:10.21037/atm-204891

4. Greco CM, Condorelli G. Epigenetic modifications and noncoding RNAs in cardiac hypertrophy and failure. Nat Rev Cardiol. 2015;12 (8):488-497. doi:10.1038/nrcardio.2015.71

5. Kuwabara Y, Horie T, Baba O, et al. MicroRNA-451 exacerbates lipotoxicity in cardiac myocytes and high-fat diet-induced cardiac hypertrophy in mice through suppression of the LKB1/AMPK pathway. Circ Res. 2015;116(2):279-288. doi:10.1161/ CIRCRESAHA.116.304707

6. Zheng D, Huo M, Li B, et al. The Role of Exosomes and Exosomal MicroRNA in Cardiovascular Disease. Front Cell Dev Biol. 2020;8:616161. doi:10.3389/fcell.2020.616161

7. He RQ, Pang YY, Zhang R, et al. Down-regulation of MiR-365 as a novel indicator to assess the progression and metastasis of hepatocellular. Int J Clin Pathol. 2017;10(9):9164-9176.

8. Huang D, Ou W, Tong H, et al. Analysis of the expression levels and clinical value of miR-365 and miR-25 in serum of patients with non-small. Oncol Lett. 2015. 20;5:191.

9. Jiang ZB, Ma BQ, Feng Z, et al. miR-365 inhibits the progression of gallbladder carcinoma and predicts the prognosis of Gallbladder carcinoma Patients. Journal of Cell Cycle. 2021;20(3):308-319. doi:10.1080/15384101.2021.1874694

10. Zhao P, Li X, Li Y, et al. Mechanism of miR-365 in regulating BDNF-TrkB signal axis of HFD/STZ induced diabetic nephropathy fibrosis and renal function. Int Urol Nephrol. 2021. doi:10.1007/ s11255-021-02853-3

11. Liu F, Zhuang L, Wu R, et al. miR-365 inhibits cell invasion and migration of triple negative breast cancer through ADAM10. J BUON. 2019;24(5):1905-1912.

12. Wang C, Su K, Zhang Y, et al. MicroRNA-365 targets multiple oncogenes to inhibit proliferation, invasion, The self-renewal of aggressive endometrial cancer cells. Cancer Manag Res. 2018;10:5171-5185. doi:10.2147/CMAR.S174889

13. Williams B, Mancia G, Spiering W, et al. 2018 ESC/ESH Guidelines for the management of arterial hypertension. The Task Force for the management of arterial hypertension of the European Society of Cardiology (ESC) and the European Society of Hypertension (ESH). G Ital Cardiol. 2018;19(11Suppl 1):3S-73S. doi:10.1714/ 3026.30245

14. Xie Y, Liu Y, Wang H, et al. Phytochrome-interacting factors directly suppress MIR156 expression to enhance shade-avoidance syndrome in Arabidopsis. Nat Commun. 2017;8(1):348. doi:10.1038/s41467017-00404-y

15. Bai C, Li X, Gao Y, et al. Melatonin improves reprogramming efficiency and proliferation of bovine-induced pluripotent stem cells. J Pineal Res. 2016;61(2):154-167. doi:10.1111/jpi.12334

16. Cui C, Wang JJ, Zhao JH, et al. A Brassica miRNA Regulates Plant Growth and Immunity through Distinct Modes of Action. Mol Plant. 2020;13(2):231-245. doi:10.1016/j.molp.2019.11.010 
17. Leimena C, Qiu H. Non-Coding RNA in the Pathogenesis, Progression and Treatment of Hypertension. Int J Mol Sci. 2018;19 (4). doi:10.3390/ijms 19040927

18. Wu H, Wang Y, Wang X, et al. MicroRNA-365 accelerates cardiac hypertrophy by inhibiting autophagy via the modulation of Skp2 expression. Biochem Biophys Res Commun. 2017;484(2):304-310. doi:10.1016/j.bbrc.2017.01.108
19. Nakamura M, Sadoshima J. Mechanisms of physiological and pathological cardiac hypertrophy. Nat Rev Cardiol. 2018;15(7):387-407.

20. Shi Y, Xi D, Zhang X, et al. Screening and validation of differentially differentlyoRNAs and target genes in hypertensive mice induced by cytomegalovirus infection. Biosci Rep. 2020;40(12). doi:10.1042/ BSR20202387

\section{Publish your work in this journal}

Pharmacogenomics and Personalized Medicine is an international, peer-reviewed, open access journal characterizing the influence of genotype on pharmacology leading to the development of personalized treatment programs and individualized drug selection for improved safety, efficacy and sustainability. This journal is indexed on the American Chemical Society's Chemical Abstracts Service (CAS). The manuscript management system is completely online and includes a very quick and fair peer-review system, which is all easy to use. Visit http://www.dovepress.com/testimonials.php to read real quotes from published authors. 